Communication

présentée le 20 mars 1964 au Comité Technique

de la Société Hydrotechnique de France

\title{
LES TYPES DE TEMPS A L'ORIGINE DES CRUES DANS LE NORD-OUEST DU MASSIF CENTRAL (1953-1962)
}

\section{PAR \\ J. PINARD *}

Le nord-ouest du Massif central possède une position privilégiée par rapport à la circulation atmosphérique générale, car ces hautes terres semblent s'avancer comme la proue d'un navire à la rencontre des nuées océaniques, mais recoivent aussi à tribord les descentes d'air arctique s'écoulant depuis la mer de Norvège, la mer du Nord et les plaines franco-belges et à babord les effluves chaudes de la Méditerranée qui se sont fourvoyées par le seuil du Lauraguais jusque sur les plateaux septentrionaux du Bassin Aquitain.

De là les «trombes » d'eau qui s'y déversent périodiquement. Que plus de $100 \mathrm{~mm}$ viennent à tomber sur une superficie de plus de $1000 \mathrm{~km}^{2}$ en $24 \mathrm{~h}$, ou en deux ou trois jours consécutifs, et ce sont de hautes eaux dans tout un bassin hydrographique, pouvant dommer des inondations sur les principaux émissaires, sinon sur tous.

En raison de ces menaces dans la répartition des crues et dans les volumes précipités en différents endroits de la région, ainsi que nous le montre la forme des isohyètes de périodes pluvieuses ou d'averses construites pour une cinquantaine de cas, il a semblé intéressant d'étudier les types de temps à l'origine de ces phénomènes en recherchant à chaque fois les conditions synoptiques qui réglaient la formation des perturbations, les caractères géographiques du type de temps et les conséquences hydrographiques que les limnigraphes enregistraient (1).

C'est un résumé de ces recherches que nous allons exposer. Ce sont trois types de temps principaux, avec certes de nombreuses variantes, qu'une cinquantaine d'exemples de hautes eaux et de crues ont permis de découvrir. Nous les appellerons les temps d'ouest au sens large, les temps du nord au sens large également et les temps du sud et leurs dérivés.

\section{LES TEMPS D'OUEST}

Ce sont les plus fréquents dans les périodes pluvieuses qui affectent nos régions, puisque nous les

\footnotetext{
* Agrégé Docteur.

(1) Nos remerciements doivent aller à MM. les Ingénieurs des Ponts at Chaussées, de l'E.D.F. et de la Météorologrie Nationale pour le précieux concours qu'ils nous ont apporté
}

avons rencontrés 27 fois sur les 45 cas étudiés. Ils se rencontrent en toute saison, mais surtout en hiver.

1. Les CENTRES D'ACTION à l'origine de ces types de temps se situent sur l'océan Atlantique et sont constitués par un centre positif plus ou moins méri-

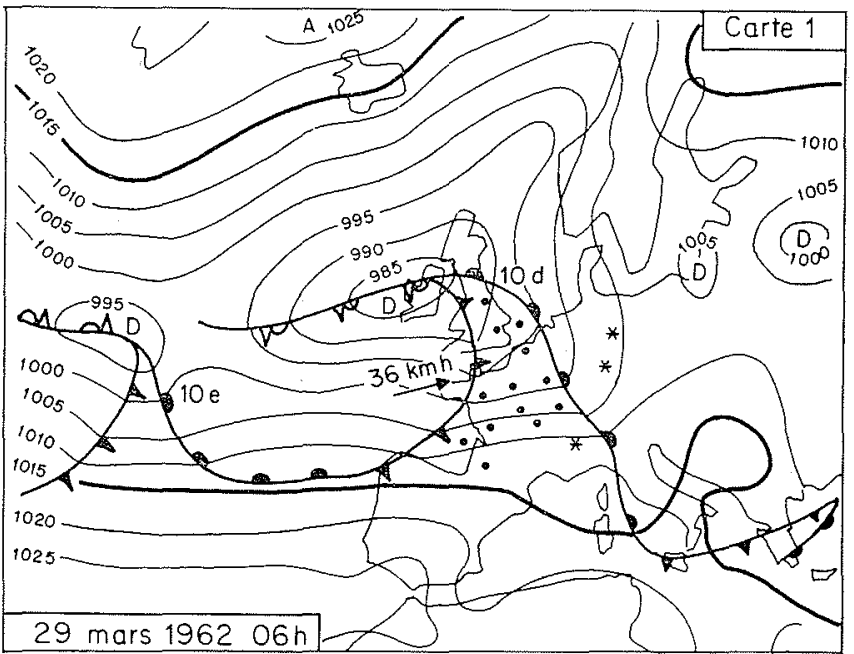

// Carte de la situation en surface.

dional : l'anticyclone des Acores ou ses prolongements, et un centre négatif nordique : la dépression d'Islande et ses dérivées. L'anticyclone des Acores peut recouvrir la Péninsule Ibérique et la Méditerranée occidentale constituant la zone d'émission des masses d'air chaud, tandis qu'au nord s'étend la zone des basses pressions atlantiques et nordeuropéennes, magnifique lit pour les perturbations qui circulent alors d'ouest en est, et surtout que des creux barométriques peuvent les attirer sur la mer du Nord, la Baltique, etc. (carte 1, 29 mars $1962,06 \mathrm{~h})$

Les conditions semblent optima pour une cyclogénèse active et prolongée, surtout si en altitude une circulation rapide de l'onde planétaire limite relativement les échanges méridiens et, de ce fait, les avancées d'air froid tant sur l'Océan que sur le continent. La circulation zonale d'ouest est alors prépondérante en altitude comme au sol et donne alors les temps doux et pluvieux, les pertur- 
bations se succèdent au rythme moyen d'une par $24 \mathrm{~h}$.

2. LES TRAITS GÉOGRAPHIQUES caractéristiques de ces temps océaniques sont le radoucissement des températures, la forte humidité relative de l'air qui se traduit par une nébulosité souvent élevée.

C'est surtout par temps d'ouest qu'ont lieu les plus fortes précipitations, surtout si une masse d'air tropical a pu accroître son humidité par un séjour prolongé sur une mer chaude (Atlantique central ou Méditerranée).

Nous avons calculé approximativement les volumes minimaux précipités (par planimétrage d'une surface inscrite à l'intérieur d'une isohyète fermée et typique). Nous obtenons, par ordre décroissant, les valeurs données par ce tableau :

\begin{tabular}{|c|c|c|c|c|}
\hline Dates & 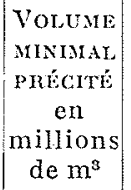 & $\begin{array}{l}\text { Maxmus } \\
\text { (en } \mathrm{mm})\end{array}$ & & $\begin{array}{l}\text { Minmun } \\
\text { (en mm) }\end{array}$ \\
\hline 2- 4 oct. 1960. & 600 & Montagne & 220 & Chabanais 26 \\
\hline $\begin{array}{c}25-28 \text { mars } 1962 \\
\text { (carte } 1 \text { bis) }\end{array}$ & 412 & Cantal & 220 & Brive 40 \\
\hline 10-13 janv. 1952. & 343 & Cantal & 250 & $\begin{array}{l}\text { Saint-Germain- } \\
\text { les-Belles } 32\end{array}$ \\
\hline 20-23 oct. 1960 & 192 & Montagne & 100 & $\begin{array}{c}\text { Saint-Yrieix- } \\
\text { la-Perche } 25\end{array}$ \\
\hline 12-15 févr. 1957 & 187 & Cantal & 225 & Saint-Junien 27 \\
\hline $9-10$ janv. 1958 & 156 & Cantal & 85 & Limoges 24 \\
\hline 20-22 nov. 1952 & 150 & Cantal & 150 & $\begin{array}{l}\text { Lussac- } \\
\text { les-Châteaux } 18\end{array}$ \\
\hline 8-10 déc. 1962. & 150 & Cantal & 155 & Nexon 25 \\
\hline 21-23 févr. 1957. & 150 & Cantal & 150 & Saint-Junien 23 \\
\hline $8-10$ oct. 1960 . & 112 & Montagne & 90 & $\begin{array}{l}\text { Availles- } \\
\text { Limousine } 13\end{array}$ \\
\hline 3- 5 déc. 1960. & 105 & Montagne & 80 & Saint-Junien 31 \\
\hline 1- 3 janv. 1952 . & 100 & Cantal & 70 & Brive 12 \\
\hline
\end{tabular}

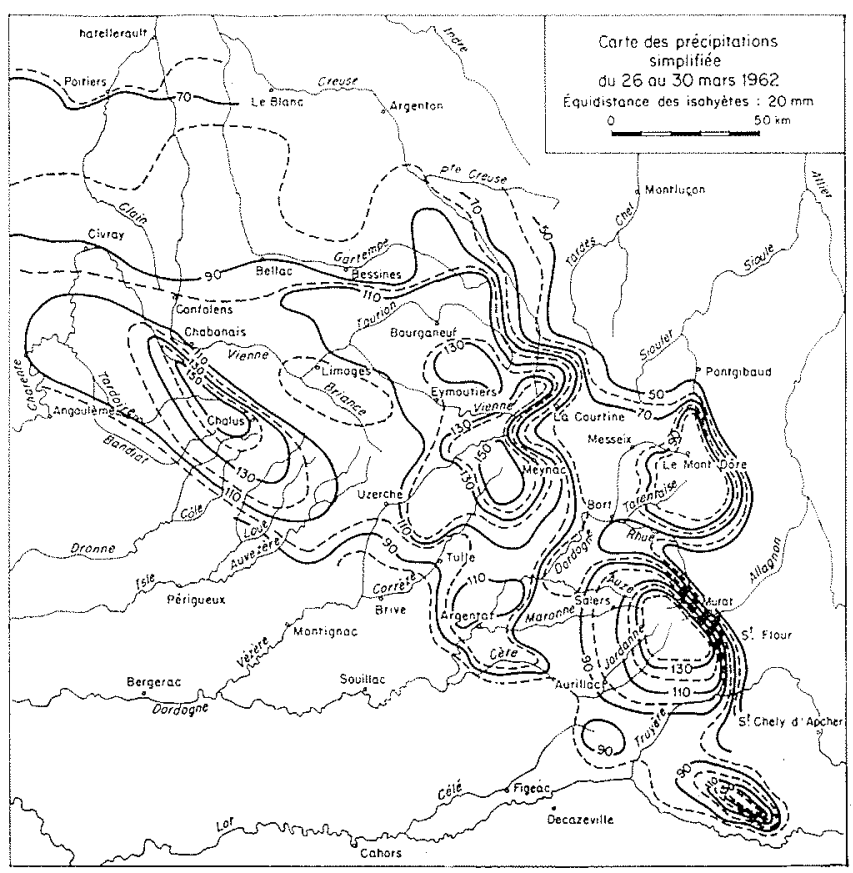

I bis/ Carte d'isohyètes.

\section{LES CONSÉOUENCES HYDROLOGIOUES.}

De telles quantités d'eau précipitées sur de vastes étendues provoquent en général de fortes crues si les sols sont saturés et au moins des crues moyennes s'ils ne le sont pas.

Si l'on étudie le rôle de ces crues à partir du volume précipité, on peut noter pour le Cher, la Vienne, la Creuse, la Vézère, l'Isle et la Dronne aux stations de jaugeages respectives de Saint-AmandMontrond, Aixe-sur-Vienne, Argenton-sur-Creuse, Montignac, Périgueux et Coutras-sur-Dronne et en fonction de ce que l'on est convenu d'appeler crues fortes et moyennes (1), les hautes eaux suivantes pour les principales averses de régime d'ouest.

\begin{tabular}{|c|c|c|c|}
\hline & $\begin{array}{l}2-4 \text { octobre } \\
1960\end{array}$ & $\begin{array}{c}27-28 \text { mars } \\
1962\end{array}$ & 9-12 janvier \\
\hline Cher & & & 3,28 \\
\hline Vienne ... & 4,00 & 3,90 & 3,55 \\
\hline Creuse . & 7,00 & $\overline{3,25}$ & 4,85 \\
\hline Vézère & $\overline{8,90}$ & 4,65 & 6,40 \\
\hline Isle ..... & 2,83 & 2,62 & 3,70 \\
\hline Dronne $\ldots \ldots$ & $\overline{3,64}$ & $\overline{3,91}$ & $\overline{5,13}$ \\
\hline
\end{tabular}

\begin{tabular}{|c|c|c|c|}
\hline & $\begin{array}{l}12-15 \text { et } 21-23 \\
\text { février } 1957\end{array}$ & $\begin{array}{c}20-22 \text { nov. } \\
1952\end{array}$ & $\begin{array}{c}8-10 \text { déc. } \\
1961\end{array}$ \\
\hline Cher & 3,70 & 3,30 & 0,49 \\
\hline Vienne & $\overline{2,80}$ & 2,70 & 2,60 \\
\hline Creuse & 3,85 & 2,95 & 2,50 \\
\hline Vézère & 6,08 & 6,40 & $5, \overline{35}$ \\
\hline Isle & $\overline{2,45}$ & & 2,43 \\
\hline Dronne & 4,49 & 5,08 & $\overline{4,07}$ \\
\hline
\end{tabular}

Par contre, après un trimestre et à plus forte raison après un semestre sec, comme le fut la fin de 1957 , les fortes averses ne suffisent pas à provoquer de grandes eaux.

\begin{tabular}{|c|c|}
\hline & $\begin{array}{l}\text { EXEMPlE APRĚs LES AVERSES DES } \\
9-12 \text { janvier } 1958\left(156 \mathrm{Mm}^{3}\right) \\
\text { et } 19-22 \text { janvier } 1958\left(81 \mathrm{Mm}^{3}\right)\end{array}$ \\
\hline Cher. . . . . . . . & $2,15(\mathrm{le} 21)$ \\
\hline Vienne. . & $1,45(\mathrm{le} 7)$ \\
\hline Creuse. . . . . . . & 2,10 (le 21) \\
\hline Vézère. . ... & $3,90($ le 11$)$ \\
\hline Isle. . .... & $1,53($ le 11$)$ \\
\hline Dronne. . ... & 2,68 (le 13) \\
\hline
\end{tabular}

\begin{tabular}{|c|c|c|c|}
\hline (1) Cotes des: & $\begin{array}{c}\text { CRues Fortes } \\
(2 \text { traits }) \\
-\end{array}$ & $\begin{array}{r}\text { Crues } \\
(1\end{array}$ & $\begin{array}{l}\text { MoYvexws } \\
\text { trait) }\end{array}$ \\
\hline Cher........... & 3,50 & & 1,50 \\
\hline Vienne.......... & 3,00 & & 1,50 \\
\hline Creuse. . . . . . . & 4,00 & & 2,00 \\
\hline Vézère.......... & 5,00 & & 3,00 \\
\hline Isle. . . . . . . . & 2,50 & & 1,00 \\
\hline Dronne......... & 4,00 & & 1,50 \\
\hline
\end{tabular}


Elles ne font grossir que quelques émissaires de bassins bien alimentés parce qu'ils ont êté le centre d'une zone plus pluvieuse comme ce fut le cas de la période pluvieuse du 26-30 mars 1962, où la zone des monts de Châlus a reçu au moins 27 millions de $\mathrm{m}^{3}$ avec plus de $110 \mathrm{~mm}$ sur une superficie de $2500 \mathrm{~km}^{2}$ environ, ce qui explique les très hautes eaux de la Vienne, grâce à la Briance $(3,90 \mathrm{~m}$ à Aixe-sur-Vienne après la confluence) et les $2,62 \mathrm{~m}$ sur l'Isle à Périgueux, principaux exutoires de ces monts du sud du Limousin, alors que par ailleurs la Creuse, la Vézère et la Dronme ne comnaissaient qu'une crue très moyenne.

Donc ces types de temps d'ouest sont à l'origine des plus fortes crues sur le nord-ouest du Massif central.

\section{LES TEMPS DU NORD}

Dans cette catégorie, on classe les types de temps dont les perturbations sont constituées par des masses d'air originaires du nord-ouest et du nord, ce qui se produit une quinzaine de fois dans les exemples étudiés.

1. LES CONDITIONS BAROMÉTRIQUES sont les suivantes :

Un anticyclone ou une dorsale s'allonge sur l'Atlantique depuis les Açores, jusqu'à la latitude de I'Irlande et dirige sur son flanc est un flux de nord-ovest ou du nord : selon la position de l'axe de ce centre de hautes pressions, le courant perturbé aura une trajectoire NO-SE ou franchement méridienne. Une dépression s'étend de la Scandinavie à la France, crée un lit de perturbations attirant l'air arctique et constitue un centre de convergence pour les masses d'air s'échappant de l'anticyclone atlantique ou d'anticyclones plus septentrionaux, comme celui du Groenland souvent associé au précédent.

Un anticyclone sur l'Europe centrale et orientale peut constituer l'autre versant de la dépression méridienne, qui peut rejoindre un creux centré sur le bassin occidental de la Méditerranée, où les descentes d'air arctique provoquent une cyclogenèse très active (carte 2, 24 mai 1958, $06 \mathrm{~h}$ ).

C'est donc un grand couloir pour la circulation méridienne qui s'établit et qui est très stable, surtout quand il est surmonté en altitude par une

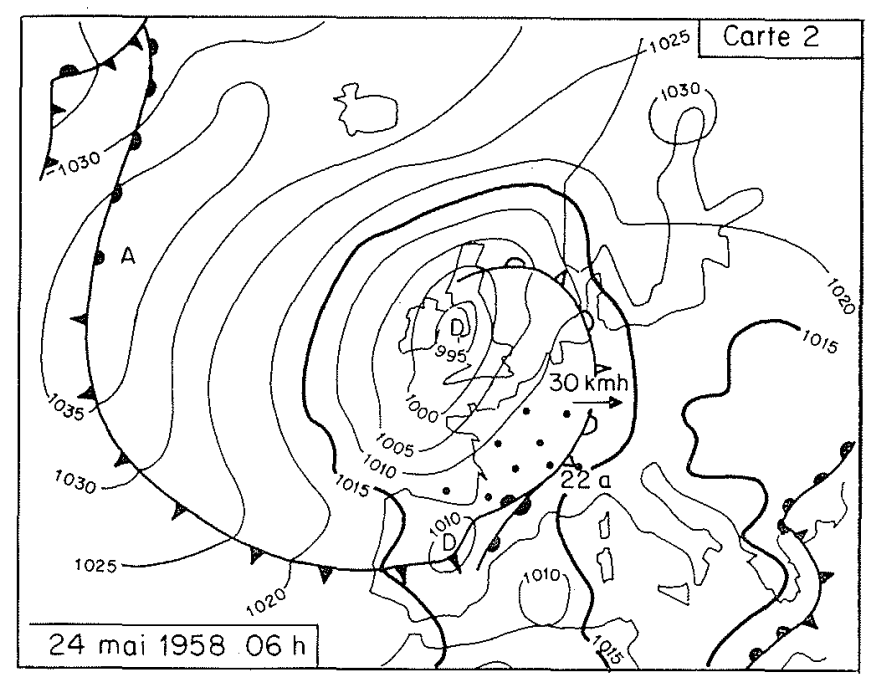

2) Carte de la situation en surface. vallée de l'onde planétaire correspondant à une circulation moyenne ou lente de cellè-ci.

2. Les efFets climatiques s'expliquent par les caractères de masses d'air. Les expulsions d'air polaire, parfois sous forme de gouttes d'air froid venant d'altilude, entraînent un rafraîchissement des températures été comme hiver et une humidité relative de l'air moins forte que dans le cas précédent, avec une succession de ciels de nuages cumuliformes et d'éclaircies. La valeur des précipitations est beaucoup plus irrégulière et leur forme varie selon les saisons : en hiver, ce sont de la neige en montagne, du grésil et en été des averses à caractère orageux; au printemps, les giboulées ont en partie cette origine. Les quantités d'eau tombées sont très variables, rarement très abondantes. Par ordre décroissant des volumes précipités, on trouve :

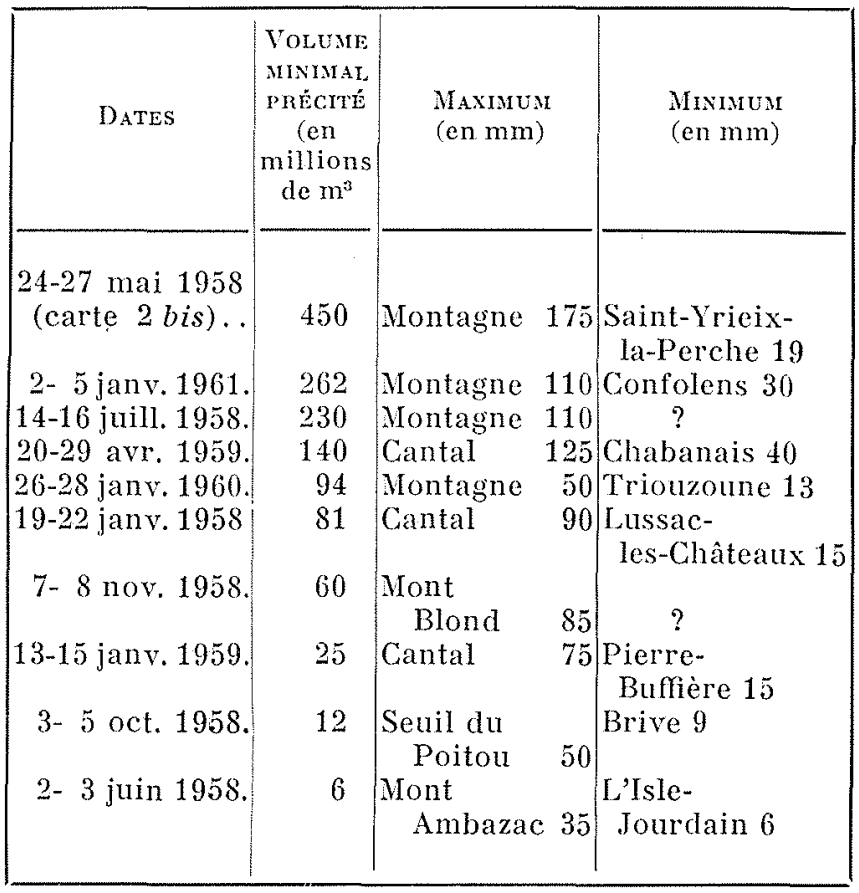

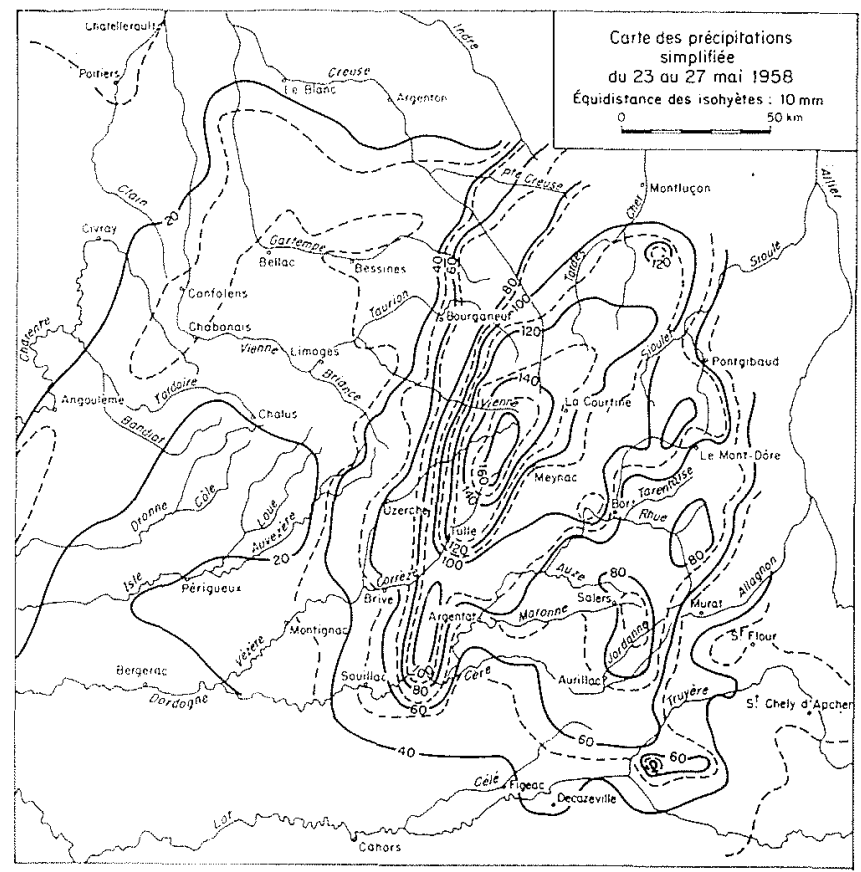

2 bis/ Carte d'isolyyètes. 
Le rapport entre maximum et minimum, dans la mesure où il présente encore une signification pour ces faibles quantités, reste toujours plus faible que dans le cas précédent.

\section{LES CONSÉQUences hydrologiQues.}

Les crues engendrées par de telles averses dépendent des temps pluvieux qui ont pu les précéder, plus encore que dans le cas précédent, où les précipitations très fortes pouvaient se suffire à ellesmêmes pour occasionner de très hautes eaux.

Donc, quand les averses de ces types de temps surviennent après des périodes de fortes pluies, on a de fortes crues, étant admis que la fonte des neiges qui peut être consécutive à ces averses est Ioin de fournir l'essentiel de l'eau de ruissellement, mais n'intervient que pour une part minime :

\begin{tabular}{|c|c|c|}
\hline & $\begin{array}{c}\text { EXEMPLE APRÈ } \\
\begin{array}{c}2-4 \text { janvier } \\
1961\end{array}\end{array}$ & $\begin{array}{l}\text { LES AVERSES DES } \\
26-28 \text { janvier } \\
1960\end{array}$ \\
\hline Cher........ & 2,94 & 2,02 \\
\hline Vienne. . . & 3,30 & $2, \overline{15}$ \\
\hline Creuse.... & 3,10 & 2,10 \\
\hline Vézère. . . . & 6,30 & 4,60 \\
\hline Isle.......... & 3,17 & 1,70 \\
\hline Dronne............ & $\overline{5,06}$ & 3,67 \\
\hline
\end{tabular}

Mais lorsque ces précipitations ont lieu sans que le sol ait été préalablement imprégné, ce sont des crues moyennes, au plus fortes sur un ou deux émissaires, qui affectent les cours d'eau.

\begin{tabular}{|c|c|c|c|}
\hline \multirow[b]{2}{*}{ Cher... } & $\begin{array}{l}\text { EXEMPIE } \\
24-27 \mathrm{mai} \\
1958\end{array}$ & $\begin{array}{l}\text { APRÈS LES AVE } \\
\mid \begin{array}{c}15-16 \text { juillet } \\
1958\end{array}\end{array}$ & $\begin{array}{c}\text { DASES DES } \\
26-30 \text { avril } \\
1959\end{array}$ \\
\hline & & 1,65 & 1,90 \\
\hline Vienne..... & 1,25 & 2,70 & $\overline{1,80}$ \\
\hline Creuse. ... & 4,00 & 3,85 & 2,10 \\
\hline Vézère. . . . & $\overline{4,62}$ & $\overline{4,50}$ & 4,75 \\
\hline Isle. . . . & & $\overline{1,52}$ & 2,36 \\
\hline Dronne....... & & & $\overline{2,78}$ \\
\hline
\end{tabular}

De ce fait, ce ne sont pas les masses d'air nordiques qui sont à l'origine de grandes crues limousines; elles provoquent surtout des débordements localisés.

\section{LES TEMPS DU SUD}

En fait, ce sont les types de temps anticycloniques et les types de temps cycloniques du secteur $\mathrm{NE}$ à $\mathrm{SE}$ que nous avons réunis sous cette rubrique, ce qui se justifie quand on envisage les centres d'action et les processus de la cyclogenèse.

\section{LEs CEnTRES D'Action.}

Ces types de temps sont avant tout caractérisés par la présence de hautes pressions sur l'Europe occidentale, centrale ou orientale, anticyclone qui diffuse sur son pourtour une mince pellicule d'air froid l'hiver, interdisant l'accès du continent aux masses d'air océaniques d'ouest ou du nord mais qui peut se laisser surmonter par des masses d'air venant du sud.

Une dépression est installée sur l'Europe méridionale ou sur le bassin de la Méditerranée et constitue un lit des perturbations qui circulent au sud de l'anticyclone septentrional et qui soumettent la France, au sud de la Loire, au flux de direction est-ouest. Les masses d'air tièdes et humides venant de la Méditerranée sont obligées de s'élever en remontant vers le nord et vont donner alors d'abondantes chutes de neige sur régions montagneuses et des pluies irrégulières sur les régions plus basses (carte 3,3 février $1959,18 \mathrm{~h}$ ).

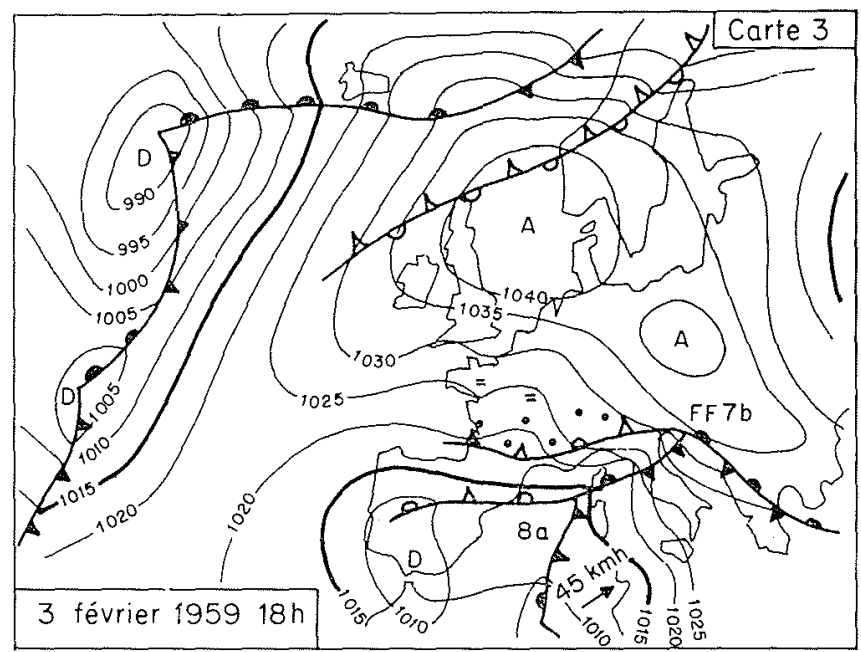

3/ Carte de la situation en surface.

Une dépression centrée fréquemment sur l'Irlande peut attirer ces masses d'air méditerranéennes qui arrivent à remonter à travers les sillons du relief convergeant vers la Méditerranée (sillon rhodanien, seuil du Lauraguais).

\section{LES EFFETS GÉOGRAPHIQUES.}

Les masses d'air d'origine continentale qui recouvrent l'Europe occidentale ont pour effet d'abaisser les températures tandis que celles qui viennent de la Méditerranée ont pour effet de les relever. Le contact des deux masses aux caractères thermiques opposés entraine des perturbations. Ces temps sont très stables et peuvent durer de plusieurs jours à plusieurs semaines pendant lesquelles les vents oscillent du NE au SE.

Les quantités d'eau tombées peuvent atteindre des valeurs assez fortes si le contraste est grand entre l'air tropical venant du sud, comme par exemple de l'air saharien humidifié, et l'air polaire ou arctique exerçant un effet de «blocking 》 pour les masses d'air océaniques qui sont obligées de le contourner.

C'est une des origines des crues cévenoles (1) particulièrement fortes aux saisons intermédiaires comme celles de septembre 1958 et peut-être de certaines crues limousines.

Les cas rencontrés ne sont pas parmi les plus

(1) N. Pardé : Le régime du Rhóne. Etude hydrologique, $2^{\circ}$ partie : La genèse des crues, 440 pages. Lyon, 1925, p. 96. 
pluvieux puisque l'on rencontre lors des averses :

\begin{tabular}{|c|c|c|c|c|}
\hline Dates & $\begin{array}{c}\text { VOLUME } \\
\text { MINIMAL } \\
\text { prÉCTE } \\
\text { (en } \\
\text { millions } \\
\text { de } \mathrm{m}^{3}\end{array}$ & $\begin{array}{l}\text { Maximuna } \\
\text { (en mm) }\end{array}$ & \multicolumn{2}{|c|}{$\begin{array}{l}\text { Mininima } \\
\text { (en mm) }\end{array}$} \\
\hline 14-16 juill. 1958 & 230 & $\begin{array}{rr}\text { Vienne } & \text { supé- } \\
\text { rieure } & 110\end{array}$ & Poitiers & 17 \\
\hline $29-30$ sept. 1958 & 218 & Montagne 95 & Le Bugue & 15,4 \\
\hline $\begin{array}{l}3-4 \text { févr } 1959 \\
\text { (carte } 3 \text { bis).. } \\
5-7 \text { janv. } 1953\end{array}$ & $\begin{array}{r}0,6 \\
\text { neige }\end{array}$ & $\begin{array}{ll}\text { Ambazac } & 17,5 \\
\text { Châlus } & 12,5\end{array}$ & Poitiers & 9 \\
\hline
\end{tabular}

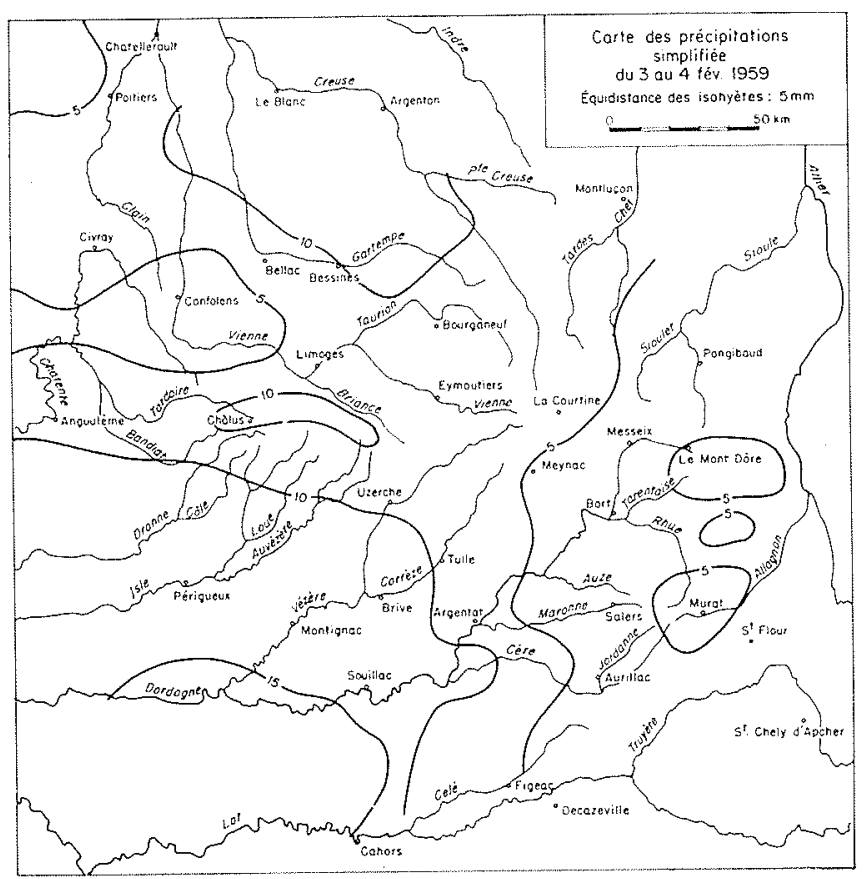

3 bis/ Carte d'isohyètes.

Mises à part les précipitations orageuses, les contrastes sont peu accusés et ce sont des pluies généralisées.

\section{Conséquences hydrologigues.}

Les hautes eaux en rapport avec les averses sont rarement importantes et sont très localisées sur quelque émissaire.

\begin{tabular}{|c|c|c|c|}
\hline & $\begin{array}{c}\text { ExwMPLE } \\
15-16 \text { juillet } \\
1958\end{array}$ & $\begin{array}{l}\text { APRÈ Y.ES AT } \\
\begin{array}{c}3-4 \text { février } \\
1959\end{array}\end{array}$ & $\begin{array}{l}\text { RSES DES } \\
\begin{array}{c}29-30 \mathrm{sept} \\
1958\end{array}\end{array}$ \\
\hline Cher. & 1,65 & 0,58 & 0,48 \\
\hline Vienne...... & 2,70 & 1,17 & 1,02 \\
\hline Creuse. . . . . . & 3,85 & 1,60 & 1,55 \\
\hline Vézère. . . . . . & 4,50 & 1,20 & 0,90 \\
\hline $\begin{array}{l}\text { Isle. . . . . . . } \\
\text { Dronne. . . . }\end{array}$ & 1,52 & $\begin{array}{l}0,54 \\
0,84\end{array}$ & $\begin{array}{l}0,50 \\
0,50\end{array}$ \\
\hline
\end{tabular}

Ces types de temps ne constituent done pas à proprement parler des occasions de crues, mais des situations qui peuvent amener des masses d'air chaud et humide à déverser de grandes quantités d'eau en quelques heures, eau qui pourra grossir les cours d'eau si le sol a été préalablement imperméabilisé

$$
\underset{* * ;}{* * ;}
$$

Ce sont donc les types de temps d'ouest qui peuvent amener les plus fortes crues, même si ce sont des masses d'air d'une autre origine qui apportent les plus forts déluges d'eau à l'origine des débordements.

Les temps du nord ont surtout pour rôle de faire intervenir des masses d'air fraîches ou froides qui vont donner des pluies modérées par opposilion aux précédentes et donc des crues dépendant de la saturation des sols, surtout pour les périodes de saison chaude.

Enfin les masses d'air d'origine méridionale peuvent donner des trombes d'eau très localisées en diverses parties du Massif central ou de ses bordures, qui grossissent alors violemment les rivieres comme c'est fréquemment le cas pour les cours d'eau cévenols et heureusement plus rarement pour les rivières limousines.

La connaissance des types de temps et de leurs conséquences hydrologiques est donc du plus grand intérêt pour la prévision des crues.

\section{Discussion.}

Président: M. Chapouthier

M. le Président remercie M. Prino de son exposé auquel le Professeur Parné, empeché, a vivement regretté de ne pouvoir assister. Il demande à M. Pinand, au sujet des «prévisions $»$, dont il apprécie la solidité, s’il a pu établir un début de bilan hydraulique pour les différents bassins.

M. Pnard indigue qu'il n'a pas calculé de bilan car', les débits n'étant pas toujours bien connus, il n'a pas osé calculer le volume écoulé et s'est contenté d'envisager le volume général précipité, sans étudier non plus la quantité d'eau qui avait pu s'infiltrer dans les sols.

M. de Rouville a remarqué que M. Prond a parlé à diver.ses reprises des couloirs de contournement des hauteurs du sud du Massif Central qu'utiliseraient les corps atmosphériques pour se propager.

On peut se demander si ces faibles barrières, en regard de l'épaisseur des gros systèmes nuageux, constituent viraiment un obstacle qu'ils essaient de contourner, déviant ainsi leur trajectoire.

Autant une chaine de montagne agit orographiouement pour augmenter localement, et surtout en deçà d'elle-mème, les précipitations, autant on la voit fonctionnant pour limiter les pluies moyennes au côté d'où soufflent les vents, autant on hésite à attribuer a des reliefs de moins de $1200 \mathrm{~m}$ un role dans la propagation des corps cylindriques importants.

Il serait d'ailleurs intéressant d'étudier ce rôle dans la réalité. Mais en a-t-on d'ores et déjà les moyens?

M. Pinard a l'impression que les systèmes nuageux sont très attachés aux incidences du relief et que môme un relief de quelques centaines de mètres tend a ctre contoumé par les systemes nuageux. On a remarqué très souvent Montagne Noire, Massif Central, vallées du Lot, de la Dordogne et dans la Limagne) l'jmportance de faibles dénivellations ou dépressions dans la direction prise par les masses d'air et les systèmes nuageux comme sur les littoraux ou audessus de grandes vallées fluviales (vallées de la Loire, de la Dordogne,... dans les Limagnes).

Les reliefs de 700 à $800 \mathrm{~m}$ et, à plus forte raison, de 1000 à $1500 \mathrm{~m}$, ont un róle sul la répartition des systèmes et sur la répartition des pluies.

M. le Professeur Chanter s'exprime en ces mots :

«Le géographe potamologue que je suis a entendu avec une satisfaction certaine la communication de $M$. Prano. Pour étudier ces types de temps sénérateurs de crues, 
M. PrNard n'a pas adopté les méthodes classiques de la climatologie (moyennes des éléments du climat) qui auraient oblitéré les caractéristiques particulières aux fortes averses; il a préféré opter ponr la climatologie dynamique, l'analyse des types de temps; c'était infiniment plus délical, étant données la complexité des phénomènes naturels, les perturbations apparemment capricieuses qui affectent le jeu des masses d'air, leur circulation, leur évolution. M. PrNaRD a eu le grand mérite de préciser la description des situations météorologiques fournies par les documents de la Météo (B.Q.R. et B.Q.E.) et de les interpréter. Après une analyse cyclogénétique bien menée, il en a étudié les conséquences géographiques et il a traduit les données pluviométriques par des cartes d'averses qu'il a commentées. Cette méthode de travail paraît valable et efficace : il a déterminé des périodes plavieuses avec effet hydrologique; il les a spatialement délimitées et il a cherché les causes du phénomène. M. PrNand a reconnu 3 types d'averses dans le nord-ouest du Massif Central : c'est un apport de qualité, peut-être trop synthétique. Et il a établi de nombreuses cartes d'averses; c'est aussi un apport de qualité. Au cours de l'examen météoro-hydrologique minutieux auquel il a procédé, il est probable que M. Pininn a pu noter des cas pleins d'intérêt qu'il n'a pu nous livrer. Certes, il ne s'agit point d'averses comparables à celles que le professeur Pandé rangerait dans «l'espèce cévenole virulente $»$, encore moins à ces trombes d'eau que M. Pardí évoquerait s'il était parmi nous et qui ont chu sur Formose, en septembre 1963, lors du cyclone Gloria : $1348 \mathrm{~mm}$ en $64 \mathrm{~h}$, dont 300 à $350 \mathrm{~mm}$ en $4 \mathrm{~h}$, de précipitations tombées sur un bassin versant de $764 \mathrm{~km}^{2}$, d'où un débit de pointe de $10300 \mathrm{~m}^{3} / \mathrm{s}$ (soit plus de $13000 \mathrm{l} / \mathrm{s} . \mathrm{km}^{2}$ ). Mais chacun sait qu'un fait capital a été établi par I'ingénicu: Delemer, au début du siècle, à savoir la réponse plus immé. diate et plus impressionnante des bassins montagneux de petites et moyennes dimensions, petites surtout, aux averses très intenses et très concentrées qu'aux précipitations continues pouvant tomber pendant plusieurs jours; un paroxysme pluvieux affectant une surface accidentée, dont les éléments de drainage convergent, provoque une pointe de crue parfois énorme et l'arrosage qui Ini succède, même s'il est digne de considération par ses effets probables dans des conditions normales, n'empêchera pas la décrue. Il me serait agréable de savoir si, dans son domaine, M. Pinard a pu constater des phénomènes semblables $\gg$ ?

M. Pinand indique que, bien que le nord-ouest du Massif Central soit la plus pluvieuse région océanique française, on est loin d'atteindre les chiffres relevés a Formose.

D'autre part, il ne semble pas évident que, dans cette région, les pluies courtes concentrées et très intenses soient plus efficaces que les pluies prolongées. Cela dépend de nombreux facteurs (saturation du sol, forme du bassin). Dans le Massif Central, Ies sols sont constitués d'une profonde arène granitique, les pentes sont en général faibles, la végétation est abondante. En fonction de ces conditions morphologiques et géologiques, il semble difficile que des averses très intenses puissent, à elles seules, gonfler suffisamment les rivières pour donner des hautes eaux ef encore moins des crues. Il faut qu'il $y$ ait saturation du sol, due en général á des périodes pluvieuses assez longues dans les jours ou semaines qui ont précédé.

Dans les cas étudiés, il a été nécessaire de dessiner des cartes d'isohyètes de 24 ou 48 heures, pour préciser le rôle des averses qui sont tombées sur la région car les diagrammes donnes par les pluviographes d'averses de 2,4 ou 6 heures ne suffisent pas à expliquer, par exemple, Ia montée d'eau d'un émissaire d'une région donnée.

M. de Beaunegarp croit que la zone d'extension des deux régimes évoqués par M. Prnard, mériterait d'être précisée dans un tableau et même, si possible, sur une carte, poul voir dans quelle mesure, éventuellement, il y aurait des zones éclaboussées à la fois par l'un et l'autre regime et, au contraire, dans quelle mesure tel régime n'affecterait qu'une région particulière.

D'autre part, peut-on déceler, selon les saisons et les mois, des periodes ou ces régimes apportent en effet des averses et des crues et d'autres où, au contraire, les crues ne sont pas très efficaces, comme l'a fait M. PARDé à propos des crues cévenoles qui se situent assez précisément du 10 septembre au 20 octobre, pour avoir le maximum d'efficacité et de grandeur si l'on considère les moyennes des situations d'une longue période.

\section{PrNard indique :}

$1^{\circ}$ qu'il est très difficile de déterminer le chevauchement dans l'espace des differents régimes météorologiques sur une région aussi étroite. Dans la mesure où une masse d'air ne peut pas être délimitée, on ne peut pas retrouver sur la carte des limites antres que les limites topographiques;

$2^{\circ}$ qu'il est évident que lorsqu'on étudie un assez grand nombre de cas, il $y$ a des périodes qui reviennent plus volontiers avec certains régimes. Dans les cas étudiés par lui, on rencontre plusien's fois des crues du début d'octobre, ou du début janvier après les pluies de la période solsticiale de la fin décembre. Par contre, on ne rencontrera pas, en février, de crues très violentes, mais l'on peut en trouver en mars avec le rétablissement progressif d'une circulation lente de l'onde planétaire, des échanges massiques méridiens de hante altitude, surtout après un hiver assez pluvieux où la circulation de l'onde planétaire a été rapide et de ce fait les échanges méridiens limités. Ce phénomène de crues serait dû alors à des types de temps du nord ou les descentes d'air arctiques sont nombreuse? et ceci jusqu'en mai-juin.

En automne, deux phénomènes différents pourraient être Ia cause de l'accroissement des précipitations :

- le maintien des situations orageuses de l'été dues à une circulation méridienne;

- l'installation de la circulation zonale d'ouest, surtout à l'origine des précipitations de saison froide.

M. Pinard n'a pu jusqu'à présent étudier que 50 cas et ne les a pas classés statistiquement dans la mesure où cette méthode ne serait pas valable sur un aussi petit nombre de cas.

M. Fontaine a beaucoup apprécié la communication de M. Pinard qui voudra bien accepter quelques remarques représentant le point de vue du météorologiste.

Retenir seulement trois types de temps à l'origine des crues dans le nord-ouest du Massif Central constitue à soll avis une simplification trop poussée. Personnellement pour des études similaires, relatives aux crues des rivieres issues du plateau de Millevaches et aux erues de la haute Durance. il ne lui a jamais été possible de retenir moins de 6 ou 7 situations types. M. CarorF, de la Division d'HydrologieE.D.F. à Chatou, dans une étude portant sur les crues de l'Isle, de la Vézère et de la Corrèze, en a retenu également six. D'ailleurs, M. Fontane pense que M. Pinari a luimême éprouvé quelques difficultés pour classer les situations étudiées dans le cadre un peu étroit qu'il s'est initialement fixé. C'est ainsi que la présence, parmi les types de temps d'ouest, de la situation météorologique a l'origine des grandes crues du début d'octobre 1960 dans le nord-ouest du Massif Central n'est pas entièrement satisfaisante, car cette situation, presque de type «cévenol $\gg$, est essentiellement caractérisée par une circulation * méridienne», de secteur sud, des masses d'air les plus humides.

Certes, presque toutes les situations météorologiques qui sont à l'origine d'importantes précipitations comportent initialement une impulsion de secteur onest, e'est-á-dire dirigée de l'océan Atlantique vers le continent européen ou vers la Méditerranée. Mais les modifications nitérieures sont telles qu'il devient évidemment nécessaire de faire des distinctions assez nombreuses.

II n'en reste pas moins que lors des grandes «averses: réputées «méditerranéennes», l'apport océanique est toujours prépondérant, surtout lorsque les masses d'air en cause sont d'origine tropicale. L'apport méditerranéen n'est que complémentaire, ce qui, d'ailleurs, n'exclut pas qu'il ait un rôle important dans le déroulement de la crua correspondante. M. le professeur Parbé, sans adopter totalement ce point de vue, a bien voulu, néanmoins, le prendre en considération.

M. Prnaro dit que dans la mesure où nous sommes dans des pays tempérés avec une circulation zonale d'ouest, tout vient de l'ouest et tout pourrait être classé ainsi.

Dans l'obligation d'être bref, M. Prxari a résumé énor mément les nombreuses variantes qui intervenaient dans l'étude de tous ces types de temps, car les centres d'action se retrouvent toujours, à l'échelle planétaire, à quelques milliers de kilomètres près. Les cas sont extrêmement nombreux et, en particulier, la crue d'octobre 1960 peut être classée dans les circulations méridiennes a partir du moment où l'on envisage la présence d'un anticyolone, sur toute l'Europe orientale, qui a pour effet de bloquer cette circulation d'ouest pendant quelque temps. Mais la question de savoir si les crues engendrées sur le nord-ouest 
du Massif Central en octobre 1960 l'ont été par des masses d'air venant de la Méditeranée on de l'océan a été très controversce dans la mesure où l'anticyclone oriental a bloqué toutes les masses d'air provenant de locéan et que ce sont surtout des masses d'air méridionales qui sont retombées à la latitude du $45^{\circ}$ ou $50^{\circ}$ parallèle.

M. Pinano pense en définitive que, au nord-ouest du Massif Central, ce sont les masses d'air océanique, quand elles entrent en conflit avec d'autres masses d'air, qui apportent les plus grandes quantités de pluie : c'est tout à fait différent sur la bordure orientale du Massif Central. M. le Président est obligé, par l'horaire, d'interrompre cette discussion, et conclut que, d'une manière générale, toute representation scientificue est forcément toujours un peu schématique, et qu'à ceriains égards, cette schématisation n'est pas pour deplaire aux hydrauliciens si, naturellement, elle correspond suffisamment à la réalité. M. le Plésident remercie à nouveau M. Pinaro.

\section{Abstract \\ Types of weather behind the floods in the North-West of the Central Massif (France) \\ by J. Pinard *}

The north-western part of the Central Massif enjoys a special position in the general atmospheric circulation pattern, as it is affected by moist oceanic air from the west, by polar air from Scandinavia and by warm air from the Mediterranean. Flood conditions due to rainfall associated with these circulations vary in extent and development. It is interesting to study the formation of these various types of weather and to relate them to the isohyetal charts for the showers and to some of the flood water levels in the distributaries in the Limousin region.

The "western" type of weather centres about an anticyclone over the Azores or Southern Europe and a depression extending from Ireland to the Baltic. It brings abundant rainfall, with contrasting peak and minimum values (see Table 1). The flood conditions it causes normally occur in autumn and winter and are usually pronounced everywhere (see Table 2).

The "northern" type of weather is caused by a ridge of high pressure extending from north to south over the Atlantic and a depression extending from Scandinavia to the Western Mediterranean and forming a centre of disturbances and southerly polar air streams. A further anticyclone sometimes also extends over Central Europe. Rainfall is of a more unpredictable type (sharp showers, thunderstorms, etc.) and may occur at any time of the year, though less abundantly than in the previous case. Floods are more local in extent and depend more on the state of soil saturation than in the previous case (see Tables 3 and 4).

The "southern" type of weather results from inflows of moist warm air masses from the Western Mediterranean which, in meeting the cooler or cold air masses centred over Northern Europe, cause heavy rainfall and major floods over the whole Central Massif. A typical case of this occured in the Limousin region in october 1960 (see Tables 5 and 6).

A knowledge of the meteorological origins of these types of weather is extremely valuable. Isohyetal charts can be of considerable use in this connection by enabling the recurrence of disastrous conditions to be predicted and appropriate counter-measures taken in good time.

\footnotetext{
* Agrégé de l'Université.
} 


\section{PARIS \\ LA CRUE DE 1910}

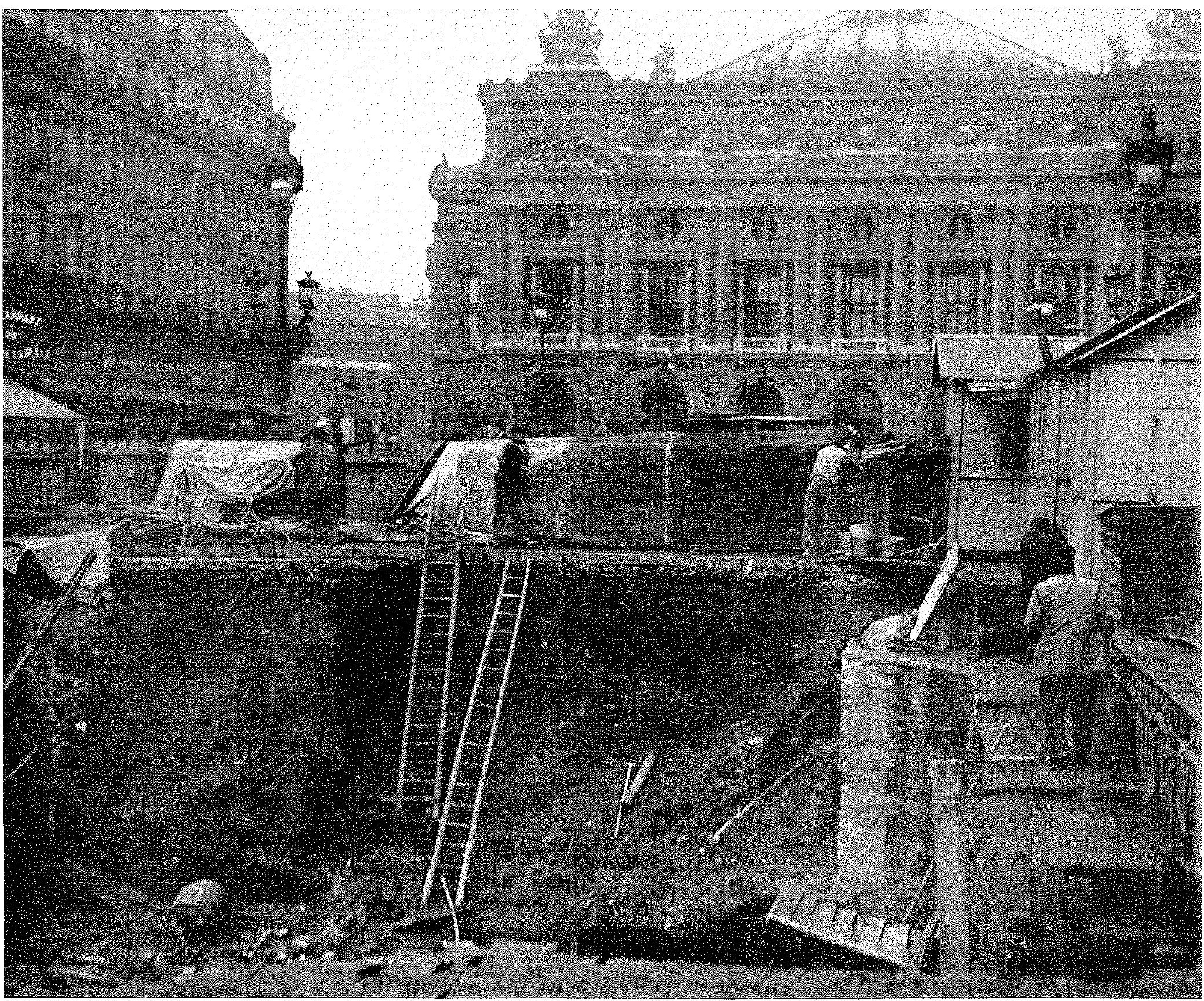

Affouillements causés par la Seine place de l'Opéra

(Photo Boyer-Viollet) 